\title{
Volume oscillation and acoustical scattering of a gas bubble
}

\author{
Yan $\mathrm{Ma}^{*}$, Tao Ma, and Feiyan Zhao \\ College of Physics and Electronic Information Engineering, Engineering Research Center of Nanostructure and \\ Functional Materials, Ning Xia Normal University, Gu Yuan 756000, China
}

\begin{abstract}
The exact solution of a gas bubble' volume was obtained based on volume oscillation of a gas bubble. The volume pulsation, acoustic impedance, scattering pressure of a gas bubble, acoustical power of scattering and acoustical scattering cross section of a single bubble are researched in a small amplitude acoustic field. The results show that a big bubble oscillates more violently than that of a small bubble in a weak acoustic field if the linear resonance does not happen. The occurrence of a linear resonance response of a single bubble leads to the volume oscillation and the scattering ability of a gas bubble become stronger. Additionally, the scattering cross section does not depend on the driving pressure. The amplitude of scattering pressure of a big bubble can reach the magnitude compared to the driving pressure when the resonance response occurs.
\end{abstract}

\section{INTRODUCTION}

Under radiation of acoustic waves, a gas bubble will oscillate. During the oscillation of a gas bubble, a diverging spherical wave can be emitted and radiation pressure can be generated within the liquids. Because of effect of bubbles, the parameters of acoustic propagation such as acoustic impedance and acoustic attenuation coefficient are changed in liquids [1]. The influence of bubbles on the acoustic propagation in liquids is mainly acoustical scattering of bubbles. So the acoustical scattering is a famous acoustic question, and causes widespread attentions [2-7]. Acoustical scattering of gas bubbles is very important for facilitating the reaction of chemical process [8-11], performing noninvasive therapy and drug delivery [12-14], and monitoring activities near ocean surface in acoustical oceanography [15]. Acoustical scattering of a gas bubble has been studied by many researchers over the past decades [16-18]. The results showed that the surface tension on the scattering coefficient of bubbles is obvious when the acoustic power is big enough. However, the above researches are based on the radial oscillation. The computation is complex and the second order small quantities of the radius are omitted in the radial analysis. Therefore the approximate values of sound parameters could not reflect the acoustical scattering of bubbles exactly. The volume oscillation equation of a small amplitude acoustic field avoids the above shortcomings. Based on the volume oscillation of a gas bubble, we can get the simple and accurate results. This is a big advantage for researching the acoustical scattering of a gas bubble in a small

\footnotetext{
${ }^{*}$ Corresponding author: manyan 2050@,163.com
}

amplitude acoustic field.

The purpose of this paper is to research the acoustical scattering of a gas bubble by using the model of a volume oscillation in a small amplitude acoustic field. Our main result is that we get the exact solution of a gas bubble' volume oscillation. The volume pulsation, acoustic impedance, scattering pressure of a gas bubble, acoustical power of scattering and acoustical scattering cross section of a single bubble are researched based on the exact solution of volume oscillation of a gas bubble. It is hoped that our results can provide a theory for facilitating the reaction of chemical process, performing noninvasive therapy and drug delivery.

\section{Mathematical model}

Periodic enforced changes in the pressure on a gas bubble result in volume oscillation of the bubble. If the amplitude of the volume pulsation is small, the motion of the bubble system is described by a second-order linear differential equation ${ }^{[19]}$, which can be shown as

$$
\begin{aligned}
& \frac{\rho}{4 \pi R_{0} \ddot{v}}+b \dot{v}+\left(\frac{\gamma P_{0}}{V_{0}}\right)\left(1+\frac{2 \sigma}{P_{0} R_{0}}-\frac{2 \sigma}{3 \gamma P_{0} R_{0}}\right) v= \\
& -P_{a} \exp \left[j \omega\left(t-\frac{r}{c}\right)\right]
\end{aligned}
$$

where $v$ is the change of volume, the dot denotes the time derivative, $\rho$ is the density of the liquid, $\sigma$ is the surface tension, $\gamma$ denotes the polytropic exponent, $R_{0}$ is the equilibrium radius of a bubble, and $b$ is the dissipation coefficient which can be described as

$$
b=2 \pi f_{0} m \delta
$$


where $\delta$ is the total damping, $m$ is generalized mass of a bubble, and $f_{0}$ is the resonant frequency of a gas bubble. $\mathrm{m}$ and $f_{0}$ are expressed as

$$
\begin{gathered}
m=\frac{\rho}{4 \pi R_{0}} \\
f_{0}=\frac{1}{2 \pi \mathrm{R}_{0}} \sqrt{3 \gamma \mathrm{P}_{0}\left(1+\frac{2 \sigma}{\mathrm{P}_{0} \mathrm{R}_{0}}-\frac{2 \sigma}{3 \gamma \mathrm{P}_{0} \mathrm{R}_{0}}\right) / \rho}
\end{gathered}
$$

The analytical solution of Eq. (1) can be expressed as

$$
v=v^{\prime} \exp \left[j\left(\omega\left(t-\frac{r}{c}\right)-\varphi\right)\right]
$$

where $v^{\prime}$ is the amplitude of volume oscillation. $v^{\prime}$ can be expressed as

$$
v^{\prime}=\frac{P_{a}}{\sqrt{\omega^{2} b^{2}+m^{2}\left(\omega_{0}^{2}-\omega^{2}\right)^{2}}}
$$

$\varphi$ is the initial phase of a bubble, and the tangent of $\varphi$ is

$$
\operatorname{tg} \varphi=\frac{\omega b}{m\left(\omega_{0}^{2}-\omega^{2}\right)}
$$

During the oscillation of a gas bubble, a diverging spherical wave can be emitted and radiation pressure can be generated within the liquids. The flow of the liquid is irrotational, therefore, a velocity potential exists. The velocity potential of a liquid particle at a distance $r$, due to a simple source, in a liquid at rest at infinity, is [19]

$$
\Phi=\frac{\dot{v}}{4 \pi r}
$$

The velocity of this liquid particle is

$$
\dot{\mathrm{r}}=-\nabla \Phi
$$

To calculate the pressure field of a single bubble, Euler equation is used,

$$
\frac{\partial \dot{\mathrm{r}}}{\partial t}+(\dot{\mathrm{r}} \cdot \nabla) \dot{\mathrm{r}}=-\frac{1}{\rho} \operatorname{grad} P_{S}
$$

where $P_{S}$ is the scattering pressure of this bubble. Combining Eq. (5), Eq. (8), Eq. (9) and Eq. (10), the scattering pressure of this bubble can be expressed as

$$
P_{S}=\frac{\rho \ddot{v}}{4 \pi r}=-\frac{\rho P_{a} \omega^{2}}{4 \pi r \sqrt{\omega^{2} b^{2}+m^{2}\left(\omega_{0}^{2}-\omega^{2}\right)^{2}}} \exp \left[j \left(\omega\left(t-\frac{r}{c}\right)-\right.\right.
$$

For presentation of the results, the amplitude of scattering pressure $\mathrm{A}$ is used, which is defined as

$$
A=\left|\frac{-\rho P_{a} \omega^{2}}{4 \pi r \sqrt{\omega^{2} b^{2}+m^{2}\left(\omega_{0}^{2}-\omega^{2}\right)^{2}}}\right|
$$

When the bubble expands and contracts periodically, the intensity of scattering sound is

$$
I_{S}=\frac{1}{T} \int_{0}^{T} \operatorname{Re}\left(P_{S}\right) \operatorname{Re}(\dot{v}) d t
$$

where Re denotes real part, and $\mathrm{T}$ is driving period. The acoustical power of scattering is

$$
\overline{W_{S}}=\iint I_{S} d S=\iint I r^{2} \sin \theta d \theta d \varphi=
$$

$$
\frac{\rho P^{2} \omega^{4}}{8 \pi c\left[\omega^{2} b^{2}+m^{2}\left(\omega_{0}^{2}-\omega^{2}\right)^{2}\right]}
$$

The acoustical scattering cross section is an important scattering parameter, which is usually employed to evaluate the scattering ability of a bubble when it is excited by the incident acoustic waves. The acoustical scattering cross section can be expressed as [20]:

$$
\mathrm{S}_{s}=\frac{\overline{w_{S}}}{I_{r}}
$$

Substituting Eq. (13) and Eq. (14) into Eq. (15), we obtain

$$
\zeta_{s}=\frac{\rho^{2} \omega^{4}}{4 \pi\left[\omega^{2} b^{2}+m^{2}\left(\omega_{0}^{2}-\omega^{2}\right)^{2}\right]}
$$

\section{Numerical results}

\subsection{Volume oscillation of a gas bubble}

The calculations were carried out with atmospheric static pressure $P_{0}=1 \mathrm{bar}$ and driving pressure $P_{a}=0.9 \mathrm{bar}$. The other parameters were set to $\sigma=0.0725 \mathrm{~N} / \mathrm{m}, \gamma=$ $1.4, \delta=0.1, \rho=1000 \mathrm{~kg} / \mathrm{m}^{3}$ and $\mu=1 \times 10^{-3} \mathrm{~kg} /(\mathrm{m}$. s).

In order to research the volume oscillation, the volume amplitude and the initial phase of a gas bubble are simulated. The main results are presented in Figs. 1 and 2, where the volume amplitude and the initial phase are shown for different driving pressures and dampings. In Fig.1, it can be seen that the volume amplitude of a gas bubble depends on the driving pressure and driving frequency. The volume amplitude in a big driving pressure field is bigger than that of a small driving pressure field when the driving frequency remains a certain value. For a constant driving pressure, the linear resonance behavior of a gas bubble is shown in Fig.1.

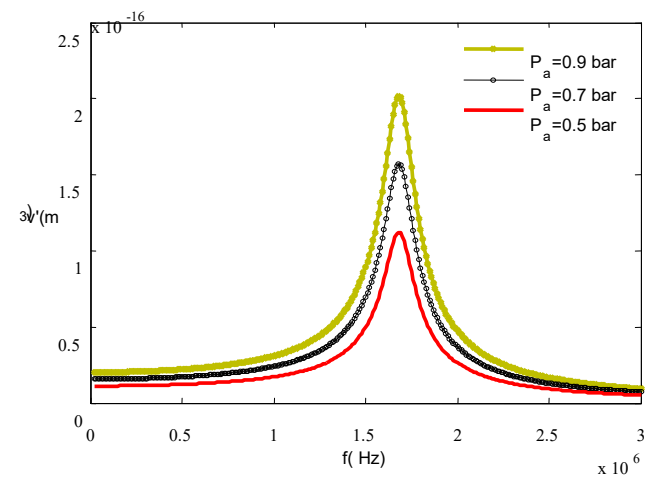

Fig. 1. Amplitude of $v$ for different driving pressures ( $f=20 \mathrm{kHz}$ and $R_{0}=2 \mu \mathrm{m}$ ).

When the driving frequency is equal to the linear resonance frequency of a gas bubble, the resonance of the bubble appears and the volume amplitude of the bubble reaches the maximum. For illustration, let us consider a section of Fig. $2, P_{a}=0.9$ bar, for a constant bubble size of $R_{0}=2 \mu \mathrm{m}$. It can be seen that if the driving frequency is much smaller (bigger) than the resonance frequency of a gas bubble, the initial phase is very small and tends to 0 . When the driving frequency is near to the linear

\footnotetext{
${ }^{*}$ Corresponding author: manyan_2050@,163.com
} 
resonance frequency, the initial phase gets to the extreme value. This result shows that the initial phase lags $\pi / 2$ when the bubble resonates. It is demonstrated that resonance has an important influence on the oscillation of a gas bubble in a small amplitude acoustic field. In addition, the damping has an influence on the initial phase of a gas bubble. It is also shown that a gas bubble oscillates more violently with a small damping in a weak driving acoustic field.

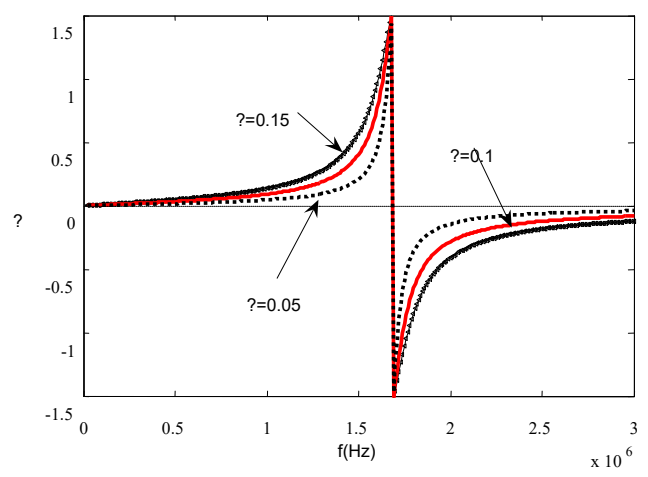

Fig. 2. Initial phase for different damping $\left(R_{0}=2 \mu \mathrm{m}\right.$ and $\left.P_{a}=0.9 \times 10^{5} \mathrm{~Pa}\right)$.

\subsection{Dissipation of a gas bubble to an acoustic field}

When a gas bubble oscillates in an acoustic field, a diverging spherical wave can be emitted and radiation pressure can be generated within the liquids. Although the radius of a gas bubble is very small (only several microns in diameter), it also has a strong sound effect compared with other suspended particles which are incompressible in liquids.

The acoustical scattering of a gas bubble in liquids is an important acoustical question. The scattering pressure, acoustical power of scattering and acoustical scattering cross section of a gas bubble are numerical simulated in a small amplitude acoustic field.

The amplitude of the scattering pressure of a gas bubble is an important parameter to evaluate the scattering ability of a bubble. The main results are presented in Fig.3, and Fig.4, where the amplitudes of scattering pressures are shown for different damping and different bubble radii. The results show that if the driving frequency is smaller than the linear resonance frequency of a gas bubble, the scattering pressure of the bubble increases with the increasing of the driving pressures. When the driving frequency is smaller than the linear resonance frequency of a gas bubble, the scattering pressure reaches a maximum value. If the driving frequency is smaller than the linear resonance frequency of a gas bubble, the scattering pressure decreases with the increasing of the driving frequency. In addition, the amplitude of scattering pressure depends on the driving pressure, radius of a gas bubble and damping. The results show that the amplitude of scattering pressure is bigger with a bigger driving pressure and a smaller damping. Especially, the radius of a gas bubble is a very important factor to the acoustical scattering.

For illustration, let us consider a section of Fig.4, $P_{a}=0.9$ bar, for a constant bubble size of $R_{0}=5 \mu \mathrm{m}$. It can be seen that when the driving frequency is equal to the linear resonance frequency of this bubble $\left(R_{0}=5 \mu \mathrm{m}\right)$, the amplitude of scattering pressure can get 0.9 bar. This result shows that the amplitude of scattering pressure of a gas bubble has a similar magnitude compared to the driving pressure when the gas bubble resonates.

Acoustical scattering cross section is usually employed to evaluate the scattering ability of a gas bubble. Therefore, the scattering cross section of a gas bubble is numerical simulated based on the volume oscillation of the bubble. The main results are presented in Fig.5 and 6, where the acoustical scattering cross sections of a gas bubble are shown for different radii and dampings.

In Fig.5, different curves resulted for the acoustical scattering cross sections of different bubbles. It can be seen that the acoustical scattering cross section of a gas bubble is very sensitive to the bubble' radius. The bigger of the radius of a gas bubble, the acoustical scattering cross section is bigger. When the driving frequency is equal to the linear resonance frequency of a gas bubble, the acoustical scattering cross section of this bubble gets to the maximum. The results show a big gas bubble has a stronger scattering ability in a small amplitude acoustic field, especially when the resonance phenomenon of a bubble appears.

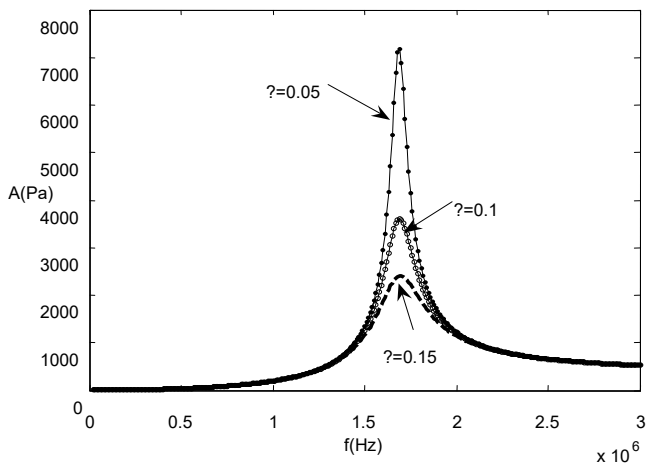

Fig. 3. The amplitude of scattering pressure for different damping $\left(P_{a}=0.9 \times 10^{5} \mathrm{~Pa}\right.$ and $\left.R_{0}=2 \mu \mathrm{m}\right)$.

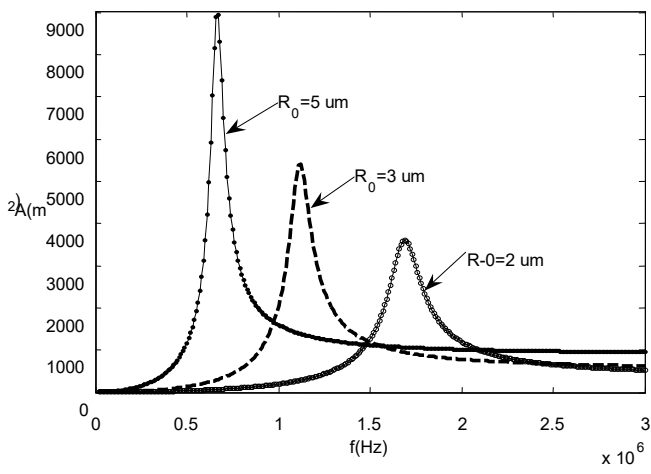

Fig. 4. The amplitude of scattering pressure for different radii $\left(P_{a}=0.9 \times 10^{5} \mathrm{~Pa}\right)$.

In Fig.6, the influence of damping on the acoustical cross section of a gas bubble is shown. For illustration, 
we consider a section of $P_{a}=0.9$ bar, for a constant bubble size of $R_{0}=2 \mu \mathrm{m}$. The different curves are results for different dampings, which are shown in Fig.6. The uppermost is obtained for $\delta=0.05$. It can be seen that the damping has a certain influence on the scattering ability of a gas bubble in a small amplitude acoustic field. When the driving frequency is much bigger or smaller than the linear resonance frequency, the acoustical scattering cross section of a gas bubble $S_{S}$ is insensitive to the bubble' radius. But when the driving frequency is equal to the linear resonance frequency of a gas bubble, the acoustical scattering cross section of a gas bubble $S_{s}$ is very sensitive to the bubble' radius. It can be seen that the acoustical scattering cross section decreases over 3 times when the damping is doubled.

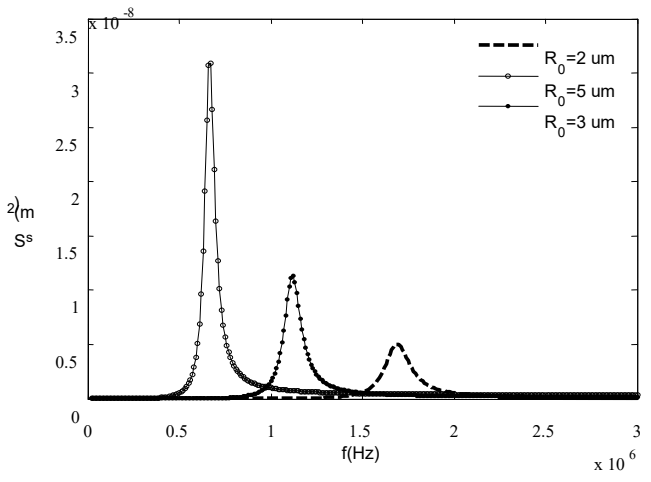

Fig. 5. Acoustical scattering cross section for different radii $\left(P_{a}=0.9 \times 10^{5} \mathrm{~Pa}\right)$.

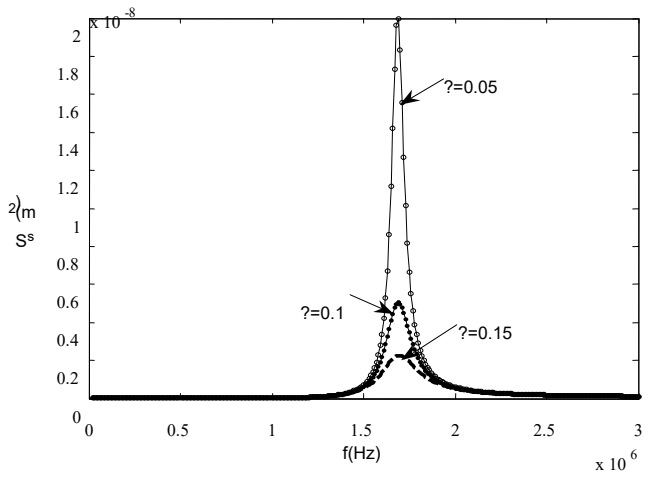

Fig. 6. Acoustical scattering cross section for different damping $\left(P_{a}=0.9 \times 10^{5} \mathrm{~Pa}\right.$ and $\left.R_{0}=2 \mu \mathrm{m}\right)$.

This result shows that the damping hinder the scattering of a gas bubble when the driving frequency is equal to the linear resonance frequency in a weak acoustic field.

\section{Conclusions}

The acoustical scattering of a gas bubble is a very important acoustic phenomenon, which can change the parameters of sound propagation in acoustic fields. The volume oscillation and the acoustical scattering of a gas bubble are researched in a weak acoustic field. The calculations have been carried out using Devin' volume equation. We get the exact solution of a gas bubble' volume oscillation. The volume pulsation, acoustic impedance, scattering pressure of a gas bubble, acoustical power of scattering and acoustical scattering ${ }^{*}$ Corresponding author: manyan_2050@163.com cross section of a single bubble in a small amplitude acoustic field $\left(P_{a}<0.9\right.$ bar $)$ are researched based on the exact solution of volume oscillation.

In a weak acoustic field, a big bubble oscillates more violently than that of a small bubble when the driving frequency is not equal to the linear frequency of the bubble. When the driving frequency is equal to the linear resonance frequency of a gas bubble, the linear resonance response of a single bubble occurs. This results lead to the volume oscillation and the scattering ability of a gas bubble become stronger. The initial phase of a gas bubble lags $\pi / 2$ when the resonance appears. The amplitude of a big bubble can get the similar magnitude compared to the driving pressure when the resonance response of this bubble occurs.

Our findings are important for the acoustical scattering of gas bubbles, the reaction of chemical process, performing noninvasive therapy and drug delivery.

The authors wish to acknowledge the Natural Science Foundation of Ning Xia of China (2018AAC03244), the Project of Higher Schools of Ning Xia of China (NGY2018-123, NGY2018-122), Ningxia key research and development program of general science and technology projects (2018BEE03026) and the Top Discipline Construction (Pedagogy) Foundation of Colleges and Universities of Ning Xia, China (Grant No. NXYLXK2017B11) for their financial supports.

\section{References}

1. O. Lousinard, Ultrason. Sonochem 19, 56 (2012)

2. H. Medwin, Ultrasonics 15, 7 (1977)

3. F. Jimenez, A. Crespo, Ultrasonics 54, 643 (2005)

4. V. Leroy, A. Strybulevych, M. G. Scanlon, J. H. Page, Eur. Phys. J. B Condens. Matter. Complex Syst 29, 123 (2009)

5. S. Hilgenfeldt, D. Lohse, M. Zomack, Eur. Phys. J. B Condens. Matter. Complex Syst 4, 247 (1998)

6. J. W. L Clarke, T. G Leighton, J. Acoust. Soc. Am 107, 1922 (2000)

7. L. Agostino, C. E. Brennen, J. Acoust. Soc. Am 84, 2126 (1988)

8. P. R. Gogate, A. B. Pandit, Rev. Chem. Eng 17, 1 (2001)

9. N. P. Vichare, P. Senthilkumar, V. S. Moholkar, P. R. Gogate, Ind. Eng. Chem. Ees 39, 1480 (2000)

10. S. P. Levitsky, Z. P. Shulman, Theoretical and Applied Rheology 26, 689 (1992)

11. H. J. Yoo, C. D Han, AIche Journal 28, 1002 (1982)

12. C. C. Coussios, R. A. Roy, Ann. Rev. Fluid Mech 40, 395 (2008)

13. G. R. Haar, S. Daniels, Phys. Med. Biol 26, 1145 (1981)

14. B. Dollet, S. M. Van, V. Garbin, et al, Ultrasound Med. Biol 34, 1465 (2008) 
15. H. Medwin, C. S. Clay, T. K. Stanton, J. Acoust. Soc. Am 105, 2065 (1999)

16. Y. N. Zhang, S. C. Li, Ultrason. Sonochem 26, 437 (2015)

17. Y. Wang, S.Y. Lin, X. L. Zhang, Sci. Sin., Ser. A 43, 917 (2013)

18. Y. Z. Fan, H. S. Li, C. Xu, Acta Phys. Sin. 66, 014305 (2017)

19. D. Charles, J. Acoust. Soc. Am 31, 1654 (1959)

20. J. Q. Huang, F. X. Li, Applied Methmatics and Mechanics 15, 549 (1994) 